\title{
Rezensionen und Kurzanzeigen
}

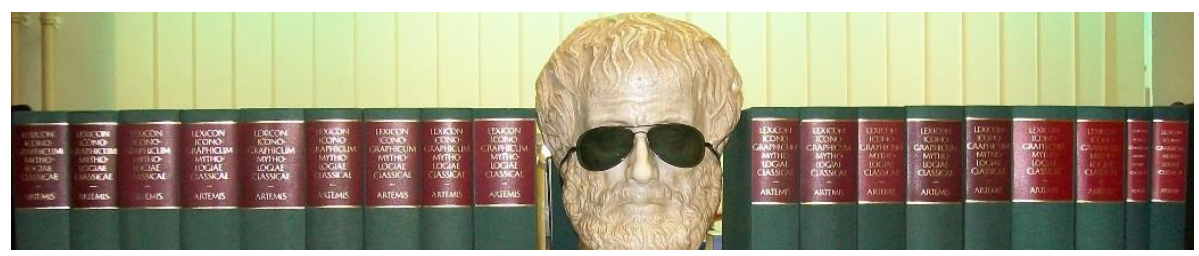

Raimund Merker: Ioanna K a r a m a n o u, Refiguring Tragedy. Studies in plays preserved in Fragments and their Reception, Berlin-Boston: Walter de Gruyter 2019 (Trends in Classics. Supplementary Volumes. 80, ed. by Francesco M o n t a n a r i - Antonios R e n g a k o s). 162 S. ISBN 987-3-11-065974-0

Sonja Schreiner: George K a z a n t z i d i s (ed.), Medicine and Paradoxography in the Ancient World. Berlin-Boston: Walter de Gruyter 2019 (Trends in Classics. Supplementary Volumes. 81, ed. by Franco M o n t a n a r i - Antonios R e n g k o s). VIII + 225 S. ISBN 978-3-11-066037-1; e-ISBN (PDF) 978-311-066177-4; e-ISBN (EPUB) 978-3-11-066047-0; ISSN 1868-4785

Sonja Schreiner: Gregor B it t o - Anna Gin e st í R o s e 11 (Hg.), Philologie auf zweiter Stufe. Literarische Rezeptionen und Inszenierungen hellenistischer Gelehrsamkeit. Stuttgart: Franz Steiner Verlag 2019. (Palingenesia. 115.) 280 S. ISBN 978-3-515-12357-0 (Print); ISBN 978-3-515-12361-7 (E-Book) 30

Sonja Schreiner: Philipp D e e g, Der Kaiser und die Katastrophe. Untersuchungen zum politischen Umgang mit Umweltkatastrophen im Prinzipat (31 v. Chr. bis 192 n. Chr.). Stuttgart: Franz Steiner Verlag 2019. (Geographica Historica. 41.) 317 S. ISBN 978-3-515-12374-7 (Print); ISBN 978-3-515-12375-4 (E-Book)

Sonja Schreiner: Robin G lin a ts i s, De l'Art poétique à l'Épître aux Pisons d'Horace. Pour une redéfinition du statut de l'œuvre. Lille-Villeneuve d'Ascq : Presses Universitaires du Septentrion 2018. (Cahiers de philologie. 34. Série Apparat critique). 203 S. ISBN 978-2-7574-2021-8; ISSN 0990-4476; ISBN 978-2-7574-2031-7 (PDF); ISBN 978-2-7574-2041-6 (ePub); ISBN 978-2-7574-2051-5 (Lot papier + numérique)

Sonja Schreiner: Christine S chmitz, Juvenal. Hildesheim: Georg Olms 2019. (Studienbücher Antike. 16.) 248 S. ISBN 978-3-487-15741-2; ISSN 1436-3526

Christoph Schwameis: Macrobius Ambrosius Theodosius. Kommentar zum Somnium Scipionis. Herausgegeben, übersetzt, erläutert und mit Indices versehen von Friedrich $\mathrm{H}$ e b e r 1 e i n. Mit einem Gastbeitrag von Christian T o r n a u. Stuttgart: Franz Steiner Verlag 2019. (Bibliothek der lateinischen Literatur der Spätantike. 1.) 478 S. ISBN 978-3-515-12365-5 
Sonja Schreiner: Carl-Friedrich B i e r i t z - Clemens Cornelius B r in k ma n n - Thomas Ha y e (Hg.), Literarische Widmungen im Mittelalter und in der Renaissance. Konzepte - Praktiken - Hintergründe. Stuttgart: Anton Hiersemann Verlag 2019. (Quellen und Untersuchungen zur lateinischen Philologie des Mittelalters. 21.) VII + 346 S. ISBN 978-3-7772-1903-5; ISSN 0721-6203

Sonja Schreiner: Christina S ch a efer - Simon Ze i s berg (Hg.), Das Haus schreiben. Bewegungen ökonomischen Wissens in der Literatur der Frühen Neuzeit. Wiesbaden: Harrassowitz Verlag 2018. (Episteme in Bewegung. 13.) IX + 300 S. Ill. ISBN 978-3-447-11074-7; ISSN 2365-5666

Nina Aringer: Andrea B e y e r, Das Lateinlehrbuch aus fachdidaktischer Perspektive. Theorie, Analyse, Konzeption. Heidelberg: Universitätsverlag Winter 2018. 419 S. ISBN 978-3-8253-6971-1

Herausgeber: Herbert Bannert - Kurt Smolak

Redaktion: Sonja Schreiner

Titelbild: Sonja Reisner

Rezensionsangebote erbeten an: klass.phil.rezensionen@univie.ac.at 
Ioanna K a r a m a n o u, Refiguring Tragedy. Studies in plays preserved in Fragments and their Reception, Berlin-Boston: Walter de Gruyter 2019 (Trends in Classics. Supplementary Volumes. 80, ed. by Franco M o n t a n a r i - Antonios Reng a ko s). 162 S. ISBN 987-3-11-065974-0

Im vorliegenden Band werden - in den vier übergeordneten Kapiteln (I. „Inter-dramatic Dialogues“; II. „Tragedy through Aristotelian Spectacles“; III. „Iconographic Reception“; IV. „Performing Fragments“) - von der Autorin eine Reihe von Fallstudien zusammengefasst, welche sich mit den unterschiedlichen Aspekten des Nachlebens verlorener bzw. nur rudimentär überlieferter griechischer Tragödien befassen, entweder in ihrer ursprünglich existierenden poetischen Form oder in ihrem fragmentarischen Zustand in späteren historischen Zeiträumen.

Ioanna K a r a m a n o u untersucht anhand der euripideischen Tragödien Alexandros (415), Alkmeon von Korinth (406/400), Diktys (431), Alope und Antigone (Aufführungsdaten unbekannt), auf welche Weise die fraglichen Dramen überarbeitet, literarisch diskutiert, dargestellt und neu aufgeführt worden sind und in welchem Maß diese die inspirative Grundlage für andere Kreativschaffende, insbesondere in der Literatur (so z. B. Menander) und Ikonographie (unteritalische Vasenmalerei, etruskische Kunst) bildeten. K a r a m a n o u argumentiert hierbei völlig richtig, dass die Erforschung der tragischen Fragmente und ihrer Rezeption nicht nur einen Einblick in das Werk selbst gewähren, sondern hierbei auch die unterschiedlichen ideologischen und kulturellen Wege und Ebenen be- und durchleuchtet werden können, auf denen die (fragmentarischen oder verlorenen) Tragödien, ausgehend vom Dichter, über Gelehrte, Künstler, Leser, bis hin zum Betrachter eines Bildes und zum Zuschauer im Theater, aufgenommen wurden.

Dass K a r a m a n o u, in Bezug auf das eben Ausgeführte, sich methodisch auf sicherem Terrain bewegt, belegt ihre langjährige Beschäftigung mit dem Thema sowie ihre daraus resultierenden Abhandlungen und Monographien (Euripides: Danae and Dictys, Leipzig-München 2006; Euripides: Alexandros, Berlin-Boston 2017). Demgemäß fügt sich ihre dritte monographische Schrift - die hier vorgestellt wird - gleichsam nahtlos an ihre vorhergehenden Arbeiten zum griechischen Drama und seiner Rezeption sowie ihre intensive Auseinandersetzung mit den euripideischen Dramenfragmenten an.

Ihr sorgfältig gemachtes Buch richtet sich vorzugsweise an einen Personenkreis aus Theater- und Literaturwissenschaftler/innen, die sich eingehender mit Fragen zur Mise-enScène, Dramaturgie und Performance des griechischen Dramas auseinandersetzen respektive den Wunsch in sich tragen, sich künftig näher mit diesem reizvollen Thema zu beschäftigen. Aber auch der ,traditionell veranlagte Klassische Philologe“ mit Interesse am griechischen Drama wird seine Freude an dem kleinen Buch haben, da K a r a m a n o u es nicht verabsäumt hat, ergiebig auf die erforderlichen Quellen und relevanten griechischen Textpassagen zu verweisen respektive diese - gerade im Hinblick auf dem im Wissenschaftsbetrieb nicht immer leicht $\mathrm{zu}$ vermittelnden Unterschied zwischen (Theater-)Praxis (Performance) und Theorie (Text) - mit passender Übersetzung abzubilden.

Ein ausführlicher Anhang (32 Seiten „Bibliographie“, „General Index“ und „Index of Passages Discussed“) rundet die gelungene Arbeit ab. Insbesondere die erschöpfende Bibliografie und die große Anzahl der besprochenen Textstellen, beginnend mit Aischylos über Aristoteles bis hin zu Varros De lingua Latina, gilt es positiv hervorzuheben.

Abträglich bzw. im höchsten Maße hinderlich für die Verbreitung des Buches außerhalb von Fachbibliotheken ist die (nach wie vor) überhöhte Preispolitik des De-Gruyter-Verlags. Ein 
Buch mit 162 Seiten und einer Handvoll s/w-Bilder für 100 Euro anzubieten trägt leider dazu bei, dass viele an dem Thema Interessierte darauf verzichten werden, sich ein Exemplar ins hauseigene Regal zu stellen.

Raimund Merker

George Kazantzidis (ed.), Medicine and Paradoxography in the Ancient World. Berlin-Boston: Walter de Gruyter 2019 (Trends in Classics. Supplementary Volumes. 81, ed. by Franco Montanari - Antonios R e n g a k o s). VIII + 225 S. ISBN 978-3-11-066037-1; e-ISBN (PDF) 978-311-066177-4; e-ISBN (EPUB) 978-3-11-066047-0; ISSN 1868-4785

Der inspirierende Band ist aus einem Kolloquium zum Thema „Medicine and Paradoxography in the Ancient World" hervorgegangen, das am 15. Dezember 2016 an der Universität Patras stattgefunden hat. Neuland betritt und „Trends in Classics“ folgt die Publikation insofern, als Medizin und Paradoxographie bisher mehr neben- als miteinander betrachtet wurden. Bereits in der nur einseitigen „Preface“ steht die „symbiotic relationship between the two“ (V) im Zentrum, in der umfänglichen „Introduction. Medicine and Paradoxography in Dialogue: Approaching thauma across Science and Fiction" (mit reichhaltiger Bibiographie ganze vierzig Seiten) erläutert der Herausgeber dieses Miteinander dann detailliert an den einzelnen Beiträgen, zeigt die schier endlose Möglichkeit an Zugängen und definiert das thaumazein mit einem eingängigen Zitat von Nita Krevans aus dem 2005 erschienenen Beitrag „The Editor's Toolbox“ (4): „the key to the genre [...] is the objective and rational presentation of an item which appears to break the laws of nature; its aim is not the satisfied 'aha!' of understanding but the round-eyed 'oh!' of wonder. “Ziel des Bandes ist der Nachweis, dass Staunen in der Wissenschaft und der Mirabilienliteratur auf vielfältige Weise seinen Platz hat. Den Nachweis führen neun Beiträger/innen: Maria G e r o l e m o u widmet sich „Technological Wonder in Herodotus' Histories" und stellt natürliche körperliche Gegebenheiten menschlicher Einflussnahme gegenüber. Katerina O i k o n o m o p o u l o u behandelt „Paradoxography and the pseudo-Aristotelian Problemata“ und regt durch ihr beständiges Gegeneinanderführen von Paradoxographie und Wissenschaft zum Nachdenken und Revidieren des eigenen und des aristotelischen Verständnisses des Paradoxen an. Floris O v e r d u i n analysiert in „In the Realm of the Two-Headed Snake: Pragmatics and Aesthetics of Mirabilia in Nicander's Theriaca and Alexipharmaca" die Funktion wundersamer Darstellungen in Nikanders Euvre und kommt zu dem Schluss, dass es ihm nicht primär auf Medizin, Pharmakologie und Biologie ankommt, sondern dass er befasst ist ,with the creation of his own exciting world, a world, that does not need to be entirely truthful, as long as it is convincing from the viewpoint of his own presentation.“ (92) Lucia F l o r i d i wendet sich in „Wondrous Healings in Greek Epigrams (and their Parodic Counterparts)“ Tempelinschriften, Posidipp und der Anthologia Graeca zu. Zur Vielfalt der thematisierten Wunderheilungen in Epigrammform (iamatika) und Prosa (iamata) gehört auch parodistische Verzerrung, die bis zur Ärzteschelte reicht. Medizin und mirabilia verbindet Julia D or o s ze w s k a in „Beyond the Limits of the Human Body: Phlegon of Tralles' Medical Curiosities“, indem sie Phlegons Schilderungen als Fallbeispiele und nicht als Prodigien einordnet, wobei der Bogen vom Scheintod über Intersexualität bis zu Mehrlings- und Interspeziesgeburten reicht. Gemeinsam ist diesen Phänomenen im Bereich der ,intersection between paradoxography and ancient medicine“ 
(137) das Aufzeigen der Grenzen des menschlichen Körpers. Grenzüberschreitungen bei Phlegon thematisiert auch Kelly E. Shannon-Henderson. Sie konzentriert sich auf dessen Kentaurendarstellung (,Phlegon's Paradoxical Physiology: Centaurs in the Peri Thaumasion“). Der Reiz dieses Beitrags liegt zum einen in der Vielfalt der unterschiedlichen Kentaurendarstellungen, was die ,genaue' Grenze zwischen Mensch und Pferd betrifft, die keineswegs immer beim Torso gezogen wird, und zum anderen in den zahlreichen Zeugnissen, weshalb die Existenz dieses faszinierenden Mischwesens physiologisch und ernährungstechnisch unmöglich ist, was die Autoren aber nicht davon abhielt, weiterhin über dieses thauma zu schreiben. Der Einsatz von thaumata zur Statussicherung und zur Abgrenzung von rivalisierenden, ignoranten Medizinern ist für Jessica Lightf o ot („Galen's Language of Wonder: Thauma, Medicine and Philosophie in On Prognosis and On Affected Parts") die Leitlinie ihrer Darstellung. Philosophie und Medizin sind untrennbar miteinander verbunden; zudem befördert Wundersames und Wunderbares das Forschungsinteresse. Heilung durch Literatur im allgemeinen und Rhetorik im besonderen, aber auch mittels Musik steht bei Georgia P e trid o u im Zentrum („Literary Remedies and Rhetorical Prescriptions in Aelius Aristides: Medical Paradoxography or Common Practise?"). Sie kann nachweisen, dass es nicht Aelius Aristides' Alleinstellungsmerkmal ist, rhetorische Übungen gegen Atemwegsbeschwerden eingesetzt zu haben. Charakterisch für ihn ist vielmehr, dass er diese Therapien auf ein neues Niveau gehoben und gleichzeitig der göttlichen Gnade besondere Heilungskraft attestiert hat. Abschließend sucht Michiel M e e u s e n theologisch begründete Antworten auf komplizierte oder sogar unlösbare naturwissenschaftliche Fragen („Unknowable Questions and Paradoxography in ps.-Alexander of Aphrodisias' Medical Puzzles and Natural Problems“"). Grundsätzlich darf göttliche Vorsehung nicht hinterfragt werden; entscheidend ist aber gar nicht so sehr, ob diese Sichtweise auf devote Religiosität gründet oder auf wissenschaftliche Unkenntnis, sondern vielmehr, ,that the implied reader [...] now has an explanatory carte blanche up his sleeve when confronted with problems he finds difficult to solve, that is, the concept of ,unsayable properties‘ (212).

Umfangreiche Literaturverzeichnisse am Ende jedes Beitrags ermöglichen weitere Vertiefung in die ohnehin schon gründlich aufbereitete Materie. Zwei Indices (zu Namen und Sachen; zu Orten) erleichtern die Zusammenschau und damit die Erreichung des gesteckten Zieles, Zusammenhänge und Abhängigkeiten (noch) deutlich(er) sichtbar zu machen. Einige Schlüsseltexte - wenig überraschend Aristoteles - kommen in mehreren Beiträgen (mit den immer gleichen Textausschnitten) vor; dies tut der Lesbarkeit und dem Gewinn, den man aus der Lektüre zieht, aber keinen Abbruch - zumal außer Rezensent/innen die meisten Rezipient/innen einzelne Beiträge und nicht das ganze Buch lesen werden, was im vorliegenden Fall besonders schade ist, da die Einzelteile, aber auch die Gesamtheit Einblick geben in das Naturund Körperverständnis des antiken Menschen. Dazu gehört auch der fehlende Respekt vor den Mitgeschöpfen: Vivisektion ist mehrfach Thema, bleibt aber stets auf das Wunderbare bei der anatomischen Demonstration beschränkt; über den tier(un)ethischen Aspekt schreibt niemand. 


\section{Gregor Bitto- Anna Ginestí Rosell (Hg.), Philologie auf zweiter} Stufe. Literarische Rezeptionen und Inszenierungen hellenistischer Gelehrsamkeit. Stuttgart: Franz Steiner Verlag 2019. (Palingenesia. 115.) 280 S. ISBN 978-3-515-12357-0 (Print); ISBN 978-3-515-12361-7

Der vorliegende Sammelband beruht auf einer Tagung (gleichen Namens) an der Katholischen Universität Eichstätt-Ingolstadt im März 2015. Abstracts (am Ende des Bandes) ermöglichen eine schnelle erste Übersicht über die breitgefächerten Inhalte. In den „Index locorum notabiliorum" sind aufgrund der Materialfülle nur die zentral(st)en Stellen aufgenommen. Zwölf Beiträge (in deutscher, englischer und französischer Sprache) verteilen sich auf zwei Großabschnitte („Dichterische Rezeptionen“ und „Literarische Inszenierungen“). Die Einleitung bietet unter Verweis auf die Abstracts (16, n. 43) nicht die ansonsten oft übliche Vorstellung der Einzelbeiträge, sondern beginnt mit der Feststellung, dass es wahrscheinlich ,der Traum eines jeden Philologen“ (9) ist, seine eigenen Arbeiten als Gegenstand literarischer Auseinandersetzung zu sehen - wofür der Band reichlich Beispiele liefert. Um den Hintergrund zu verstehen, folgt ein Abriss zur „Entstehung der ,klassischen“ Philologie“ (19), ein weiterer zur „römischen Rezeption“ (12) und schließlich „Literarisierung in der Kaiserzeit“ (14) und eine knappe „Konzeption des Bandes“ (16) mit angeschlossenem umfänglichen Literaturverzeichnis. Genres greifen durchwegs ineinander: So schreibt Nunzia C i a n o darüber, was passiert, „Wenn der exegetische Auxiliartext zum dichterischen Text wird. Über Ciceros Benutzung der Scholien zu Arat". Cicero wird zum hellenistischen Dichter römischer Schule, der auf eine ,enge Verbindung zwischen Dichtung und philologischer Exegese in den Aratea“ (35) setzt. C i a n o sieht in der „Untrennbarkeit des griechischen Gedichts von seinem exegetischen Apparat von Kommentaren und Scholien [...] das Bedürfnis der lateinischen Übersetzung, gleichfalls auf eben dieses exegetische Material zurückzugreifen“ (35). Es wird zum „interpretamentum, das den Vorgang des vertere anleitet" (35). Als Parallelbeispiele führt sie die Odusia, die Medea des Ennius, die Aeneis und die horazischen Oden an. Jean-Christophe J o live t stellt sich (und seinen Leser/innen) die Frage „'Pourquoi Cyréné n'a pas plongé?' L'embuscade de Protée et la philologie homérique (Virgile, Géorgiques 4, 387-530 et les scholies à l'Odyssée)“". Er kann den Nachweis führen, dass Vergils Homerphilologie keine sterile Gelehrsamkeit, sondern Frucht eines gelehrten Horizonts ist, den der Dichter in Reaktion auf Kommentare und grammatici Graeci mit seinem Publikum teilt. Joan P a g è s beleuchtet „Perseus: The Mythographic Tradition and its Reception in Ovid's Metamorphoses" und weist nach, dass Ovid bei aller Inspiration durch griechische Modelle seine kreative Umsetzung auf literarisches Wissen und vergleichende Forschung baute, auf ein Zusammenspiel von Literatur und Philologie, unter Einbeziehung von Epik, Tragödie und Mythographie. Als poeta doctus war ihm an einer seltene(re)n Variante gelegen, mit der er seinen Lesern Neues und Überraschendes bieten konnte. Eine Forschungslücke möchte Chiara B att is te 11 a in „Seneca tragicus and the scholia to Euripides. Some case studies from the Medea" schließen und aufzeigen, wie sich Senecas Benützung von exegetischem Material zu Euripides auf seine Stücke ausgewirkt hat. Sie kommt zu dem Ergebnis, dass bei Seneca das gesprochene Wort über der Gestik und der szenischen Gestaltung steht. Gregor B i t t o fokussiert auf das kaiserzeitliche Epos und Homer (,Sed plura vacant. Statius' Achilleis und die Homerphilologie“) und konstatiert, dass jeder, der nach Homer und Vergil epische Dichtung über Achill schreiben wollte, sich ,mit den zwei einschüchterndsten literarischen Größen der beiden Weltliteraturen 
konfrontiert" (93) sah und entsprechend darauf reagieren musste. B i t t o führt den Nachweis, dass die Homerkommentierung eine unverzichtbare Voraussetzung für das richtige Verständnis der Qualitäten der Achilleis ist und dass Statius ,mithilfe der Homerphilologie seine Unabhängigkeit von Homer bzw. dem Homerbild der Scholien“ (113) unter Beweis stellt, was ihm nicht zuletzt durch einen „spielerischeren Umgang“ (113) als bei Vergil gelingt; während Homers Achill ein fertiger Held ist, ist der des Statius noch im Reifungsprozess, „der mehr Facetten zeigen kann" (114).

Philipp W e i $\beta$ analysiert - ohne Bezug auf Martin Walser - den „Tod eines Kritikers: Zur Zoilosanekdote bei Vitruv. 7 praef. 8-9 und ihrem Nachleben in den Saturnalia des Macrobius“. In klaren Schritten kann der Verfasser nachweisen, wie die negative Färbung des Zoilos sich im Zuge der römischen Rezeption und Nachwirkung immer mehr steigerte, bis ein geradezu klischeehafter Kritikaster aus ihm wurde. Auf jeweils einen Autor und dessen Euvre - wiederum ganz unterschiedlichen Genera zugehörend - konzentrieren sich die verbleibenden sechs Beiträge: Bardo Maria Gauly („Seneca: Von Philologie zu Philosophie“) erweist Senecas Philosophie als innovativ, so neuartig, dass sie deutlich mehr ist als reine Vermittlung griechischer Vorbilder: Er macht Philosophie durch Verbindung mit römischen exempla fruchtbar - in der Literatur und für das reale Leben. Thomas S c h i r r e n (,Philologia ancilla rhetoricae. Leseübungen für die rhetorische Brillanz? Quintilians philologische Empfehlungen“) wählt einen hochtheoretischen Ansatz und reflektiert einleitend über „Philologie der Philologie“ (147) und „Metaphilologie“ (147). Weit über Quintilians Institutio oratoria und mögliche „Leselisten“ (159) hinausreichend greift er in die griechische Antike zurück und auf die deutsche Literaturgeschichte (insbesondere Lichtenberg und Schlegel) aus. Gemeinsam ist ihnen das Hinarbeiten auf „eine neue Poesie, die durch das System ermöglicht werden soll“ (180). Richtiges Urteil(en) - iudicium - ermöglicht Kanonbildung. Anna Gi n e s tí R o s e 11 („Etymologie beim Wein. Philologie in der Gruppenidentitätsbildung der Quaestiones Convivales von Plutarch“) kommt zu dem Schluss, dass das Telos der ,inszenierten Gespräche“ (194) der Weg ist und nicht das Ziel: Es geht um die Suche nach Antworten, nicht um das Finden (195): „Durch Beherrschung komplexer etymologischer Methoden zeigen die Teilnehmer der Symposien Plutarchs nicht nur ihre Zugehörigkeit zur griechischen Elite, sondern auch die eigene Sonderstellung innerhalb dieser Gruppe.“ Ein Anhang (Text und Übersetzung) ermöglicht den Nachvollzug der Argumentation in idealer Weise für geübte und weniger geübte Leser/innen. Für Wytse K e u l e n (,Mark Aurel, der Philologenkaiser. Die Literarisierung der Philologie in Frontos Korrespondenz") ist ,'Philologie auf zweiter Stufe' ein zentraler Bestandteil der Bildungs- und Freundschaftsinszenierung beider Korrespondenten“ (201). Der Brief wird zum „Medium für die Inszenierung einer exklusiven Freundschaft“ (201), die Korrespondenz selbst wurde aufgrund der schwierigen Überliefungslage und der falschen Einschätzung, dass es sich um Privatbriefe handelt, in der Forschung vernachlässigt. Im Fokus der Betrachtung stehen Selbstdarstellung und Dialogizität. Sprachgewandtheit, asymmetrische Kommunikation und Politik mit sprachlichen Mitteln sind die bestimmenden Faktoren. Verteidigung mittels philologischer Argumente rücken bei Ute T is c he r („Der Sophist als Philologe. Inszenierung und Instrumentalisierung der grammatica in Apuleius' Rede Pro se de magia") ins Zentrum; damit gewinnt sie einem ohnehin schon singulären Text eine weitere Facette ab: Philologie inszeniert sich als Philosophie, ihr Auftritt ist ebenso forensisch wie literarisch: Die Entlarvung und Entmachtung der unwissenden Gegner funktioniert auf eine ähnliche Weise wie in den Noctes Atticae des Gellius. Die geistreiche Umwertung, der einfallsreiche Twist liegt darin, dass Apuleius’ Gegner „ungebildete Provinzler“ (250) sind, bei 
Gellius aber geistlose grammatici. Peter von Mölle nd orff (,Sub iudice philologia. Zur Verarbeitung philologischer Themen im Werk Lukians") widmet sich den Verbindungslinien zwischen Philologie, Rhetorik und Poetik. Dafür bietet sich Lukian als eine Art idealtypische ,philologische Spielwiese‘ an, insbesondere, um Philologie als agonistische Disziplin zu erweisen, deren diskursive Relevanz sich erst im praktischen Lebensvollzug und unter ständiger Anpassung an das jeweilige Gegenüber entfaltet. Erst dann ist Philologie kein „totes Wissen“ (270) mehr - ein ideales Schlusswort für ein vielgestaltiges und abwechslungsreiches Buch, das auch Skeptiker/innen von der Wichtigkeit der ,Liebe zum Wort', zur Philologie, gleichsam reduziert auf ihre ,DNA', überzeugen sollte, ist sie doch in allen Lebenslagen einsetzbar situationsadäquat und wandelbar wie der weiter oben bereits genannte Proteus.

Sonja Schreiner

Philipp Deeg, Der Kaiser und die Katastrophe. Untersuchungen zum politischen Umgang mit Umweltkatastrophen im Prinzipat (31 v. Chr. bis 192 n. Chr.). Stuttgart: Franz Steiner Verlag 2019. (Geographica Historica. 41.) 317 S. ISBN 978-3-515-12374-7 (Print); ISBN 978-3-515-12375-4 (E-Book)

Katastrophenmanagement ist zu einem Dauerthema in der Medienlandschaft des 21. Jahrhunderts geworden - gelungenes ebenso wie katastrophal danebengegangenes. In der Druckfassung seiner Stuttgarter Dissertation zeigt Philipp D e e g, dass die Bewältigung und der Umgang mit traumatisierenden Naturereignissen, Seuchen, Hungersnöten und von Menschen verursachten folgenschweren Unfällen wie Bränden ihren Niederschlag in der antiken Literatur und in der Wahrnehmung der jeweiligen Herrscher als Katastrophenmanager, aber auch in deren Selbstdarstellung gefunden hat. Nach einleitenden Begriffsdefinitionen („Naturkatastrophe“, „Sozialkatastrophe“, „Umweltkatastrophe“) wendet sich der Verfasser dem Prinzipat als politischem System, in dem Maßnahmen ergriffen (oder verabsäumt) werden, zu, um schließlich seine Quellen (aus diversen Genres) vorzustellen. Den Hauptteil des Buches nimmt eine „Chronologische Analyse“, geordnet nach Dynastien und einzelnen Kaisern, ein, wobei Galba, Otho und Vitellius als „ephemere Kaiser“ kategorisiert werden. In einem zweiten, deutlich kürzeren, aber sehr aufschlussreichen Teil liefert D e e g eine „Strukturhistorische Analyse“, in deren Verlauf er „Hilfsmaßnahmen“ und „Präventivmaßnahmen“ bespricht. Interessant und bemerkenswert ist, dass er glaubhaft machen kann, dass die Umsetzung und Durchführung vorbeugenden Katastrophenschutzes deutlich hinter dem technischen know how der Römer zurückblieb. Dies hatte herrschaftstaktische Gründe, hätte ein Kaiser, der solche Projekte forciert, doch eingestanden, dass seine gottgleiche Soter-Funktion doch nur eine enden wollende war. In diesem Zusammenhang ist auch „Deutungshoheit“ ein wichtiges Thema, galten Umweltereignisse doch als omina. Ein dritter, ebenso knapper Abschnitt ist der „Kommunikation“ gewidmet, worin D e e g erstaunlich schnelle Abläufe, Kommunikationskanäle und „Medien des Danks“ (wie z. B. Münzen) vorstellt. Zwei Anhänge runden die innovative Arbeit ab: „Das Rhodos-Beben um 227 v. Chr. - ein kurzer Exkurs in hellenistische Zeit in vergleichender Absicht" zeigt die Wurzeln römischer Katastrophenhilfe, und eine umfangreiche, mit vielerlei Quellen, Daten und sogar Unsicherheitsmarkierungen versehene „Liste von Umweltkatastrophen 31 v. Chr. bis 192 n. Chr.“ ermöglicht eine rasche und vergleichsweise niedrigschwellige Orientierung. Wer bisher der Meinung war, Katastrophenhilfe im alten Rom sei in erster Linie mit Titus anlässlich des Vesuvausbruchs $79 \mathrm{n}$. Chr. zu 
verbinden, und dann könne man bestenfalls noch Neros Verhalten beim Brand Roms $64 \mathrm{n}$. Chr. anführen, wird von D e e g mit einer ganzen Fundgrube konfrontiert. Dass er Erdbeben immer wieder „Schütterereignisse“ nennt, tut der Qualität des unter enormem Materialaufwand entstandenen Buches keinerlei Abbruch. Vielmehr ist es Philipp D e e g s Verdienst, einen wichtigen Beitrag nicht nur zur Kulturgeschichte, sondern auch zum besseren Verständnis der Propagandamechanismen im Prinzipat geleistet zu haben. Althistoriker/innen können ebenso aus dem Vollen schöpfen wie Numismatiker/innen und Philolog/innen, denen eine Fülle von Texten, Kontexten und Interpretationen geboten werden. Diesen multidisziplinären Ansatz muss eine Qualifikationsschrift erst einmal leisten.

Sonja Schreiner

Robin G li n a t s i s, De l'Art poétique à l'Épître aux Pisons d'Horace. Pour une redéfinition du statut de l'œuvre. Lille-Villeneuve d'Ascq : Presses Universitaires du Septentrion 2018. (Cahiers de philologie. 34. Série Apparat critique). 203 S. ISBN 978-2-7574-2021-8; ISSN 0990-4476 ; ISBN 978-27574-2031-7 (PDF); ISBN 978-2-7574-2041-6 (ePub); ISBN 978-2-75742051-5 (Lot papier + numérique)

In einem systematisch entwickelten Dreischritt macht sich Robin G li in a t s i s auf die Suche nach einer neuen Definition der Ars poetica des Horaz, die er weniger als römisches Pendant zur Poetik des Aristoteles sehen will, als vielmehr - in Rückbesinnung auf das Genre, dem der Text eigentlich zuzuordnen ist - als poetischen Brief über Dichtung. Dabei kommt der feinsinnige Interpret stufenweise zu Erkenntnissen, die einen Mehrwert für das dichterische (Selbst)verständnis des augusteischen Dichters mit sich bringen. In einer konzisen „Introduction" lässt der Verfasser die Forschungsgeschichte Revue passieren und behandelt dabei neben den Fragen von Titel und Datierung besonders die Wirkunsgsgeschichte (von der Antike bis in die Gegenwart, und das stets mit dankenswert aussagekräftigen Rezeptionsbelegen und Qualitätsurteilen). Warum er Ad Pisones gegenüber Ars poetica den Vorzug gibt, erläutert er im ersten Abschnitt „L'Ars poetica confrontée aux traités antiques“, indem er sich zunächst den Charakteristika antiker Poetiktraktate zuwendet, die Bezüge zwischen Dichtung und Rhetorik beleuchtet und in weiterer Folge rhetorische Züge in der Ars poetica herausarbeitet. (Auffällig und methodisch aufschlussreich ist, dass Glin a t s is die Titelvariante, die er eigentlich ablehnt, solange verwendet, als er sein Beweisziel noch nicht vollständig erreicht hat.) Nähe und Distanz zwischen Aristoteles und Horaz folgen in einem nächsten Schritt, um schließlich das System in der Ars poetica selbst zu beleuchten und damit den Übergang zum zweiten Großabschnitt zu schlagen („Le caractère épistolaire du texte“), in dem auf einen kurzen Eröffnungsabschnitt zur Definitionsgeschichte von Briefliteratur in der Antike die Anwendung dieser Kriterien auf Ad Pisones folgt. (Ab jetzt hat Gl in a t s i s den Titel - passend zu seiner Darstellung - gewechselt.) Folgerichtig ist eine nähere Beschäftigung mit den Adressaten, wozu eine Verortung derselben im Text ebenso gehört wie ihr Einfluss auf den horazischen Diskurs. Im dritten und letzten Teil („L'épître aux Pisons, un poème sur l'art de la poésie“) fokussiert der Verfasser auf die Rollen von Tragödie und Satyrspiel in Ad Pisones und wendet sich poetischen Spiel(erei)en im Text zu, um zu dem Schluss zu kommen, dass poetische Theorie und Praxis bei Horaz nicht voneinander zu trennen sind, dass es ihm insbesondere in dem Brief, 
den Robin Gli n a t s i s ins Zentrum seiner luziden Ausführungen gestellt hat, nicht nur um Dichtung per se, sondern auch um Vermittlungsarbeit geht.

Sonja Schreiner

\author{
Christine S ch mitz, Juvenal. Hildesheim: Georg Olms 2019. (Studien- \\ bücher Antike. 16.) 248 S. ISBN 978-3-487-15741-2; ISSN 1436-3526
}

Zur bereits etablierten Reihe „Studienbücher Antike“ steuert Christine S c h m i t z mit ihrem Band zu Juvenal eine wertvolle Ergänzung bei. Das Buch besticht durch seine ponderierte Mischung aus angenehm zu lesender Einführung in das Genre, übersichtlicher Vorstellung der Inhalte und Schwerpunkte der einzelnen Satiren und detaillierter Textinterpretation, die durchaus in die Tiefe geht. Die breite und gut nachvollziehbare Diskussion der persona-Theorie (im Umfang von zwanzig Seiten) am Anfang des Buches - noch vor der Vita des Autors, literarischen Reflexen bei Martial, Gedanken über mögliche Berührungspunkte zwischen Werk und Leben und Überlegungen zur Abfassungszeit - bleibt stark in Erinnerung und unterstützt beim Verständnis der restlichen Publikation, was gerade bei einem doppelbödigen Genre wie der Satire, das von Hyperbolik ebenso ,lebt“" wie von Subtexten, in hohem Maße hilfreich ist. So ist es auch stimmig, dass der erste größere Teil, der Literaturtheorie und Autorenbiographie umfasst, mit „Der Satiriker Juvenal inner- und außerhalb seines Textes“ überschrieben ist. In einem zweiten, geringfügig kürzeren Teil stehen „Juvenal und die Gattung der römischen Verssatire“ im Mittelpunkt, ein Kapitel, in dem die Autorin Gattungsgeschichte und Juvenals Spezifika geschickt miteinander verbindet. Den Hauptteil des Buches (etwa neunzig Seiten) widmet S c h m i t z Einzelinterpretationen - sechzehn an der Zahl. Sie ist bemüht, sich allen Satiren mit vergleichbarer Ausführlichkeit zuzuwenden, und wählt dabei programmatische Titel für jede Einzelvorstellung, die den Rezipient/innen bereits einen ersten Hinweis auf den Inhalt geben. So begnügt sie sich nicht damit, die erste Satire als „Programmsatire“ zu bezeichnen, sondern erweitert den in der Forschung etablierten Terminus durch den vielsagenden Zusatz „Satire als literarisches Manifest“. Das Kapitel zur vierten Satire betitelt sie unter Anspielung auf ein gängiges Sprichwort (und den Nachkriegsroman Der Fisch beginnt am Kopf zu stinken des Österreichers Fritz Wöss [eig. Weiss], der noch bekannter ist für den Vorgängerband Hunde wollt ihr ewig leben) mit „Die Fischsatire oder ,Der Fisch stinkt vom Kopf her““. Die Vorgänge in der fünften Satire könnten kaum besser zusammengefasst sein als mit „Ein asymmetrisches Gastmahl“ - und Ähnliches ließe sich für die verbleibenden dreizehn Texte sagen. Zahlreiche Textauszüge (im Original und in Übersetzung) geben einen lebendigen Eindruck von Juvenals Sprache, Stil und Aussagewillen und machen auch Neulinge auf dem Gebiet der satura neuund begierig auf mehr. Vor- und Rückverweise auf wiederkehrende Motivik ergeben ein abgerundetes Gesamtbild. Vertiefung erfährt all dies in zwei weiteren Kapiteln: „Juvenals satirisch analysierender Blick auf die römische Gesellschaft“ und „Juvenals virtuose Technik: Satirisierung durch Sprache und Vers“. Das sechste und letzte Kapitel behandelt „Juvenals Überlieferung und Rezeption" und behandelt Interpolationen (anhand aussagekräftiger Beispiele) ebenso wie Nachwirkung (incl. Übersetzungen und Imitationen), wobei S c h m i t z den Bogen von der (Spät)antike bis ins 21. Jahrhundert spannt: Durs Grünbein steht gleichberechtigt neben dem Asterix-Band Le Papyrus de César, und auch auf Harry C. Schnurs Juvenal-Supplement zur 16. Satire vergisst sie nicht. Eine umfangreiche Bibliographie und ein Stellenregister sind nützliche Hilfsmittel des (zufällig auch!) 16. „Studienbuchs Antike“, das Juvenal-Einsteiger/innen ebenso dienlich sein wird wie Kenner/innen des Autors; erstere werden die 
kompakte Vorstellung dessen, was wichtig ist zum Verständnis der nicht immer einfachen Texte, zu schätzen wissen; letzteren wird gefallen, wie gut es Christine $\mathrm{S} \mathrm{c} \mathrm{h} \mathrm{mit} \mathrm{z} \mathrm{gelungen}$ ist, aus der Fülle dessen, was zu Juvenal publiziert wurde, das herauszufiltern, was eine ideale Basis für eine gute Vorlesung zur römischen Satire ist. Quintilians oft zitiertes dictum bekommt damit eine neue Bedeutungsebene; denn nach der Lektüre dieses Buches wird jede/r sagen können: satura tota nostra est.

Sonja Schreiner

Macrobius Ambrosius Theodosius. Kommentar zum Somnium Scipionis. Herausgegeben, übersetzt, erläutert und mit Indices versehen von Friedrich He b e r l e i n. Mit einem Gastbeitrag von Christian T or n a u. Stuttgart: Franz Steiner Verlag 2019. (Bibliothek der lateinischen Literatur der Spätantike. 1.) 478 S. ISBN 978-3-515-12365-5

Diese höchst ansprechend gestaltete Übersetzung zu einem der bekanntesten Texte der römischen Spätantike stellt den ersten Band einer neuen Reihe dar, der „Bibliothek der lateinischen Literatur der Spätantike“, deren Zielsetzungen die Herausgeber Alexander Arweiler (Münster) und Bardo Maria Gauly (Eichstätt-Ingolstadt) zu Beginn in einem Vorwort erklären. Mit dem vorliegenden Band gelingt Friedrich He berle i n, Honorarprofessor in Eichstätt, eine würdige Eröffnung.

Eingeleitet wird der Band von Christian T o r $\mathrm{n}$ a u, der seit seiner Dissertation zu Plotins Enneaden 6, 4-5 (1998) als einer der bedeutendsten deutschsprachigen Experten für den Neuplatonismus gelten darf. In seinem knappen Essay behandelt er zunächst den Autor und reiht dessen Kommentar in den neuplatonischen Diskurs ein (11-14), ohne jedoch auf Ciceros Somnium Scipionis als Werk sui generis näher einzugehen: Er widmet sich hingegen vorwiegend (14-26) Macrobius' Auseinandersetzung mit der Seele, in der er ,den gedanklichen Kern" des Werkes sieht (14). Dabei betont T or n a u die Originalität des Macrobius: Dieser habe nicht nur als Erster einen lateinischen Text zum Gegenstand eines philosophischen Kommentars gemacht $(11,26)$, auch der im Gegensatz zu der Platon und Aristoteles harmonisierenden Tradition stehende, teilweise genuin ,antiaristotelische“ Zugang könnte Macrobius selbst und nicht dessen Quellen zuzuschreiben sein (23-26). Zum Abschluss beschäftigt sich To r $\mathrm{n}$ a u mit den von Macrobius genannten Autoritäten und kommt auf dessen Haltung zum Christentum zu sprechen (26-30). Nicht behandelt werden hingegen wichtige andere, jedoch nicht oder kaum vom Autor erwähnte Quellen wie etwa Ps.-Jamblich oder Numenios von Apameia, deren Bedeutung $\mathrm{He}$ b e r le i n in seinen Anmerkungen unterstreicht. Unerwähnt bleibt leider auch die doch so entscheidende Wirkung des Textes auf das Hochmittelalter und die Renaissance.

Im Anschluss folgt eine Inhaltsübersicht (33-36), deren Kapitel sich in der Übersetzung als Überschriften in teilweise recht freier Entsprechung wiederfinden (s. u.). Der lateinische Text und die deutsche Übersetzung nehmen den Hauptteil des Bandes ein (38-365). Der Lesetext basiert auf der Belles Lettres-Ausgabe von Mireille A r m i s e n - M a r c h e t t i (2011), von der er sich in sechzehn im Anhang (377) aufgelisteten Einzelfällen unterscheidet; diese werden in den Anmerkungen jeweils begründet. Angaben zur Überlieferung finden sich nicht. Zwar legt Heberle i n hier die erste deutschsprachige Übersetzung vor, zahlreiche Übertragungen in andere Sprachen sind jedoch gerade im letzten Jahrzehnt erschienen: ins Italienische durch 
Moreno Neri (2007), ins Spanische durch Fernando Navarro Antolín (im Literaturverzeichnis 377 falsch als „Antolí“ angegeben), ins Französische durch ArmisenM a r c h e t t i. Aus den letzten Jahrzehnten stammen die zweibändige italienische Übersetzung von Mario R e g a 1 i (mit Kommentar 1983, 1990) und die englische von William Harris S t a h 1 (1966). H e b e r l e i n berücksichtigt aber, wie seine Anmerkungen zeigen, die meisten dieser Übersetzungen.

H e b e r le in s Übersetzung wirkt nicht nur sehr durchdacht, wie einige ausführliche Begründungen in den Anmerkungen zeigen, sie ist auch insgesamt stilistisch elegant und gut lesbar. Wenn auch teilweise Fachbegriffe wie das in der lateinischen Bibel verwendete Wort „Luminare“ (Plural: luminaria)“ (z. B. 57) für lumen (Plural: lumina), gemeint sind Sonne und Mond, oder „emanieren“ (z. B. 79) für defluere ungebräuchlich oder allzu gehoben wirken, so passt dies immerhin zur altertümlich-philosophischen Diktion. Hingegen existiert weder ein Wort „gelängen“ (137) für producere noch ist es verständlich: Ich würde ,in die Länge ziehen“ oder ,ausdehnen“" vorschlagen. Ich beschränke mich daher im Folgenden auf Einzelheiten: Eine möglichst genaue Parallelität im Druck zwischen dem Lesetext und der Übertragung sollte ein Anliegen jeder Übersetzung sein, um lästiges Blättern zu verhindern. Dass diese angesichts der Kürze des Lateinischen nicht immer Wort für Wort erreicht werden kann, versteht sich von selbst. Es sollte aber vermieden werden, dass Text und Übersetzung ganzer Paragraphen dergestalt getrennt werden, wie es bei 1,6,31 geschieht (80-82). He berle in entscheidet sich dazu, Zitate sowohl im Text als auch in der Übersetzung nicht, wie üblich, durch Kursivierung, sondern durch Anführungszeichen kenntlich zu machen. Wenn ganze Verse zitiert werden (58, 86, 120, 174, 190, 256, 290), verzichtet er jedoch im Lesetext auf Anführungszeichen, sondern beschränkt sich auf den Zeilenabstand und die Einrückung. Hierbei kommt es zu einigen Fehlern im Druck: 120 wird das Zitat nicht von Macrobius' Text abgegrenzt (1,9,9), 190 folgt nicht, wie überall sonst, ein Zeilenabstand $(1,18,15)$ - kurz zuvor fehlt auch ein Spatium zwischen uicinus und Tauro - und auf 290f. wird auch die deutsche Übersetzung eingerückt und ohne Anführungszeichen angegeben, was nirgends sonst der Fall ist $(2,8,1)$. Bekanntlich sind in den Handschriften auch Illustrationen erhalten, auf die Macrobius für seine Argumentation zurückgreift. Diese werden im Anhang (367-371) abgebildet; zudem wird auf sie unter dem lateinischen Lesetext verwiesen. Betreffen die Abbildungen mehrere Paragraphen, so erfolgen diese Verweise stets am Ende, was besonders bei Abb. 7 problematisch ist (2,7,4-6), da der Text hier besonders stark auf die Illustration Bezug nimmt, der Verweis jedoch erst auf der nächsten Lesetextseite erfolgt (284). Hier wäre es sinnvoll, schon hinsichtlich 2,7,3 darauf zu verweisen, wenn Macrobius die pictura einführt; in diesem Fall wäre auch Anm. 83 in der Übersetzung überflüssig.

Den Anhang beginnt $\mathrm{Heberlein}$ mit einer ausführlichen Identifizierung des Autors (373-376): Hier stellt sich die Frage, wieso diese Informationen nicht in die Einleitung integriert werden konnten? So wirkt das Kapitel wie eine ausführlichere Wiederholung des bereits Bekannten. Den Großteil des Anhangs bilden jedoch wertvolle Anmerkungen (383466). Diese beschränken sich nicht nur auf Hinweise oder Querverweise auf weitere Stellen des Kommentars oder andere antike Werke; die lateinischen Stellen werden dabei immer im Original und in Übersetzung zitiert - ein Fehler findet sich in Anm. 106 (2. Buch), 450, wo in der Übersetzung von Cic. Nat. Deor. 2,118 der erste Satz des lateinischen Zitats ausgelassen ist. Die Anmerkungen, welche teilweise fast zwei Seiten lang sind, nennen auch stets die jeweiligen Quellen der einzelnen Kapitel, hinterfragen die inhaltliche Gestaltung kritisch, berücksichtigen die Rezeption der einzelnen Stellen im Mittelalter und zeichnen sich insgesamt 
durch eine genaue Kenntnis der aktuellen Sekundärliteratur aus, die vollständig angegeben wird. Heberlein erklärt seine klugen Entscheidungen hinsichtlich des Textes oder der Übersetzung stets nachvollziehbar. Warum er aber hier erneut - freilich mit einer wiederum anderen Strukturierung und Betitelung - den Inhalt der Paragraphen zusammenfasst, der doch ohnehin im Inhaltsverzeichnis nachzulesen ist, erschließt sich mir nicht. Insgesamt wirkt der Anhang auch etwas überladen und unstrukturiert: Dass der „Dank“ an dessen fünfter Stelle, zwischen „Antike Autoren in den Anmerkungen“" und Abbildungsverzeichnis, versteckt ist, entbehrt jeder inhaltlichen Logik und schmälert dessen Bedeutung. Warum stehen das Literatur- und Abbildungsverzeichnis voneinander getrennt, warum sind sie überhaupt Teil des Anhangs? Dass im Verzeichnis der in den Anmerkungen genannten antiken Autoren deren jeweilige Editionen zitiert werden, erscheint überflüssig. So praktisch schließlich die Indices (467-478) zu antiken Autoren, griechischen Wörtern, Namen und Sachen auch sind, ist ihr Aufbau doch etwas merkwürdig: Warum wird etwa der Abschnitt „Autoren“ von dem Abschnitt „Namen“ getrennt, obwohl im letzteren u. a. deren Werke genannt werden?

Die angesprochenen Kritikpunkte schmälern jedoch insgesamt nicht den überaus positiven Gesamteindruck des Bandes, der deutschsprachigen Leser/innen, welche nicht die im Moment sicherlich führende Ausgabe von A r m i s e n - M a r c h e t t i verwenden möchten, den Zugang zu einem der Grundtexte des lateinischen Mittelalters erleichtert.

Christoph Schwameis

\section{Carl-Friedrich Bieritz - Clemens Cornelius Brinkmann - Thomas} $\mathrm{H}$ a y e (Hg.), Literarische Widmungen im Mittelalter und in der Renaissance. Konzepte - Praktiken - Hintergründe. Stuttgart: Anton Hiersemann Verlag 2019. (Quellen und Untersuchungen zur lateinischen Philologie des Mittelalters. 21.) VII + 346 S. ISBN 978-3-7772-1903-5; ISSN 0721-6203

In der konzisen Einleitung erörtern die Herausgeber zunächst die Forschungslage, um dann „Konzepte - Praktiken - Hintergründe“ zu beleuchten, drei Begriffe, denen die in aller Kürze vorgestellten dreizehn Beiträge zugeordnet werden. Carmen Cardelle de $\mathrm{Hartmann}$ eröffnet den Band mit „Grammatik als Gabe. Aldhelm, Bonifatius, Expossitio latinitatis (Anonymus ad Cuimnanum)“. Die Verfasserin legt gesteigerten Wert darauf, dass alle von ihr behandelten Zueignenden neben den üblichen Topoi ihre Rolle als Grammatiker ebenso in den Fokus rücken wie ihr Selbstverständnis als Lehrer. Die Texte haben insofern Ausnahmecharakter als sie Prioritätsanspruch erheben, Kritik an Autoritäten üben oder Grammatik verteidigen und nicht der Bibellektüre unterordnen, sondern vielmehr mit der Exegese verbinden. Ähnlichkeiten ergeben sich aus direkter Kenntnis, Selbstbewusstsein resultiert aus der kompetenten Aneignung einer fremden Sprache, die nun sie selbst, die ehemaligen Adepten, an die nächste(n) Generation(en) weitergeben. Franziska S c h n o o r (,'Minimum vilissimumque codicellum vestrae celsitudini consecrare praesumo.' Bescheidenheitstopoi in Dedikationen des frühen Mittelalters“) analysiert auf Basis von vier Autoren (Walahfrid Strabo, Wandalbert von Prüm, Milo von Saint-Amand und Notker Balbulus) das (Un)gleichgewicht von Bescheidenheit und Stolz, versucht eine Häufigkeitsstatistik der beliebtesten Topoi zu generieren und zu ermitteln, welche Rückschlüsse der Einsatz von modestia auf die Beziehung von Zueignendem und Widmungsträger zulässt. Clemens Cornelius B rinkman n nimmt den Verfasser des ersten Geschichtswerks über die Normandie in den Blick („Dudos Dedikationen. Formen und 
Funktionen der Widmung in der Historia Normannorum des Dudo von St. Quentin“). Dudo stellt einen ganzen Komplex von Widmungen voran, die abhängig vom Adressaten tatsächlich auf den Wunsch nach Lektüre abzielen oder rein panegyrisch-herrschaftslegitimierende Tendenz haben. Eine Übersicht über den mehrteiligen Prolog (79) erleichtert den Einstieg in ein Bündel von Texten, das von Dudo als Statussymbol für die Normannen angelegt ist. Thomas $\mathrm{H}$ a y e beleuchtet „Dedikationsstrategien bei Franco von Lüttich (gest. nach 1083)“ und weist Rückbezüge auf Plinius maior, Vegetius und Vitruv als Modelle nach. Diese Fachschriftsteller bieten sich an, da Franco von Lüttich De quadratura circuli verfasste. Die Elaboriertheit seiner Widmung spricht dafür, dass er nicht nur Dank für stattgehabte Förderung abstatten, sondern sich weitere Aufträge sichern wollte. Signifikant seltener als lateinische Widmungen sind im Mittelalter solche in der jeweiligen Volkssprache. Bernhard T e u b e r wendet sich englischen und französischen Beispielen zu (,'Dedicationem non semper omisit scriba vernaculus' Zum prekären Status von Widmungen in der volkssprachlichen Literatur des Mittelalters“). Antikebezüge sind immer wieder präsent, etwa wenn ein Widmungsträger Alexander den Großen mühelos übertrifft. Der Abdruck von dankenswert viel Text eröffnet den Einblick in bislang den meisten mittel- und neulateinischen Philolog/innen eher verborgene Literatur. CarlFriedrich B i e r it z (,Gerhoch von Reichersberg: Literatur, Widmung und Kirchenpolitik im 12. Jahrhundert") wechselt in den klerikalen Bereich und macht sich auf die Suche nach der Funktion von Zueignungen in diesem Kontext. Er erkennt rhetorische Muster und den Kampf um Autorität zwischen Reich und Kirche. Als Desiderat spricht der Verfasser den Vergleich mit anderen Autoren desselben Zeitraums an. In einem ausführlichen Anhang (177-186) sind die deutschen Übersetzungen der Vorreden zu De investigatione Antichristi abgedruckt, die zum tieferen Verständnis wesentlich beitragen. Susanna F i s c h e r kombiniert Widmung und Enkomiastik (,Dedikation, Patronage und Panegyrik in der Historia de duabus civitatibus, den Gesta Friderici und im Ligurinus"). Sie spürt dem literarischen Konzept der Patronage in divergenten Genres nach und spannt den Bogen von erwiesenem Mäzenatentum bis zur nur erhofften Unterstützung. Jürgen W olf stellt eine Frage, die besondere Neugierde auf seinen Beitrag wecken soll: ,Dedikation ohne Dedikation? Überlegungen zur Widmungspraxis in der volkssprachig-deutschen Literatur der Blütezeit (1160-1230)“. Er kommt zu dem Ergebnis, dass die hochstehende Themenwahl und das erlesene Figurenrepertoire die eigentliche Widmung gleichsam ersetzte (229): „Solche Werke einem Fürsten oder einer Fürstin dedizieren, scheint untadelig, und es scheint genauso untadelig, sie in Empfang zu nehmen - sogar in einem illiterat-höfischen Umfeld. Eines umfänglichen Dedikationstextes, eines Dedikationsbriefes oder gar eines Dedikationstraktates bedurfte es dazu freilich nicht - noch nicht." Bis es so weit sein wird, werden weitere 200 Jahre vergehen. Erst dann wird eine Übernahme aus der lateinischen Praxis erfolgen; und mit Christian Heitzmanns Beitrag „Cui bono? Widmungen als ,Networking‘ am Beispiel von Florentiner Humanisten des frühen 15. Jahrhunderts" ist der Bereich der neulateinischen Literatur erreicht. Der Verfasser widmet sich Leonardo Bruni, Poggio Bracciolini und Giannozzo Manetti und zeigt an ihnen „exemplarisch“ (244) nahezu alle Facetten von Erwartungen, Problemlagen und Erfolgen im Umgang mit Patronen. Die breite Palette an möglichen Widmungsträgern wird im Anhang (245-246) zu Leonardo Brunis Widmungsempfängern deutlich. Karl En e n kel steuert mit „Die rituelle Verfasstheit der Buchübergabe und ihre legitimierende Wirkung im humanistischen Literaturbetrieb des langen 15. Jahrhunderts“ einen grundlegenden und programmatischen Beitrag bei. Rituelle Aspekte der Dedikationspraxis sorgen für Legitimation und Autorität. Mit einer Analyse von Bild- und Textelementen führt er diese wirkungsvolle Methode eindrücklich 
vor Augen. Der performative Akt entscheidet über den gewünschten Erfolg; das gilt für etablierte Autoren ebenso wie für Neueinsteiger (267): „Die Textverfasser gaben sich große Mühe, durch eine optimale Instrumentalisierung des Widmungsempfängers einen möglichst ,glatten“ Übergang des von ihnen verfassten Textes in den Öffentlichkeitsraum zu gewährleisten. Dazu entwickelten sie ein feinmaschiges paratextuelles Gewebe, das sich gleichwohl zur Gänze auf gewisse Kerne einer rituellen rhetorischen Verfassung zurückführen lässt. Kreativität und rituelle Abwicklung gehen dabei [...] Hand in Hand." Die eigene Rolle als Autor und diejenige seines Adressaten steht in „Diskurs und Adressierung. Beobachtungen zur Funktion von Widmungen in den monastischen Netzwerken des Spätmittelalters“ von Marc-Aeilko A ri s im Fokus. Damit geht eine Selbststilisierung des Autors als Prophet und Lehrer ebenso einher wie das Seelenheil des Widmungsträgers und der Leser gesichert ist. Nicht um Heils- sondern um Lokalgeschichte und ganz pragmatisch-praktische (Förder)aspekte von Dedikationen geht es Bernd P o s s e $1 \mathrm{t}$ in „Zur Praxis der Förderung und Dedikation von Literatur in Augsburg und Nürnberg (2. Hälfte des 15. Jahrhunderts)“. Das lokale Element als bestimmendes wird nicht zuletzt an der „Wahl lokaler Themen und einer bilingualen Anlage“ (312) deutlich. Die „exemplarisch vorgestellten Werke von Meisterlin, Celtis und Schedel“ geben „durch eine günstige Überlieferungslage mehr Einblick in die historische Praxis der Literaturförderung und -dedikation [...], als dies bei den meisten anderen Werken üblich ist.“ Bei Bernd Roling laufen abschließend die Fäden zusammen; er verbindet mittel- und neulateinische Literatur in „Die Dedikation mittellateinischer Literatur in der editorischen Praxis der Frühen Neuzeit. Drei Beispiele aus der mittellateinischen Epik“. An Walter von Chatillôns Alexandreis, Joseph of Exeters Sex libri de bello Trojano und Gunther von Pairis' Ligurinus erläutert der Verfasser, wie Dedikationen die Aufmerksamkeit auf die vernachlässigten Texte lenken konnten und welche Editions- und Wirkungsgeschichte die als typisch mittelalterlich klassifizierte Alexandreis, der als neulateinisches Epos gehandelte und erst spät(er) als mittellateinisches identifizierte „Trojanische Krieg“ und der (all)zu perfekte und daher als Fälschung enttarnte Ligurinus hatten. Ein „Register der Personen- und Ortsnamen“ hilft bei der Orientierung in einem Feld, dessen Proponenten sich vielleicht nicht allen Leser/innen prima vista erschließen, aufgrund der reizvollen Vielgestaltigkeit ihrer Hervorbringungen aus den Bücherschränken in Hinkunft aber nicht mehr wegzudenken sein werden - ein Verdienst der Beiträger/innen dieses schönen Bandes.

Sonja Schreiner

Christina Schaefer - Simon Zeisberg (Hg.), Das Haus schreiben. Bewegungen ökonomischen Wissens in der Literatur der Frühen Neuzeit. Wiesbaden: Harrassowitz Verlag 2018. (Episteme in Bewegung. 13.) IX + 300 S. Ill. ISBN 978-3-447-11074-7; ISSN 2365-5666

Bereits der Titel des vorliegenen Episteme-Bandes verspricht etwas Außergewöhnliches. Der ungewohnte Ausdruck im Haupt- und im Untertitel lässt auf spannende (und innovative) Beiträge hoffen, und diese Erwartung wird nicht enttäuscht - ganz im Gegenteil. Hervorgegangen ist der Sammelband aus dem Sonderforschungsbereich 980 ,Episteme in Bewegung. Wissenstransfer von der Alten Welt bis in die Frühe Neuzeit“. Fünf Großkapitel verteilen sich auf etwa 275 Seiten und bieten zwölf Beiträge. Dazu tritt eine umfangreiche Einleitung der Herausgeberin und des Herausgebers und ein Abbildungsverzeichnis. Jedem Beitrag ist ein Li- 
teraturverzeichnis beigegeben. Im ersten Abschnitt („Soziale Beziehungen im Haus“) informieren Rüdiger S c h n e 11 über „Concordia im Haus - Vielfalt der Diskurse (1300-1799)“ und Anita T r a n i n g e r über „Herr und Knecht. Inszenierungen des Verhältnisses zur Dienerschaft bei Lando, Montaigne, Alfieri und Larra“. Teil 2 („Haus und Geschlecht“) umfasst drei Beiträge: Margarete Zi m merman n berichtet „Von monastischen und weltlichen ,Häusern“. Denkformen des ,Hauses“ im Werk der Christine de Pizan“. Doris R u h e behandelt „Zählen und Erzählen. Weibliche Ökonomie in den Fabliaux“, und Christina S c h a e f e r zeigt „Weibliche prudentia auf der Bühne. Zur Inszenierung ökonomischer Konzepte in Paolo Caggios Flamminia prudente (1551)“. Im dritten Abschnitt (,Haus und Staat“) geht es bei Claudia $\mathrm{O}$ p i t z - B e l a k h a 1 ,,Vom oikos zum ménage. Antikerezeption und -revision in Jean Bodins Six livres de la République (1576)“, bei Michael L o r b e r dagegen um „Hofökonomie als Biopolitik. Johann Joachim Bechers ,Werkhauß'-Projekt (1664-1683)“, das einen geographischen und konzeptuellen Bogen von München bis Wien schlägt. Kapitel 4 („Haus und Markt") thematisiert - ganz auf das Theater, Theatralizität und den untrennbaren Zusammenhang mit der gesellschaftlichen Wirklichkeit konzentriert - „Verrückte Welt. Das Ende der Ökonomik im Theater Shakespeares und Middleton" (Verena Olejniczak Lobsien), „Eifersucht und Oeconomia im englischen Theater der Frühen Neuzeit“ (Anne E n d e r w i t z) und „Ökonomisches Wissen auf der Bühne. Die Komödie und der Übergang von der alteuropäischen zur modernen Ökonomik“ (Daniel Fulda). „Subversive Schreibweisen“ sind das verbindende Element der letzten beiden Beiträge, die Kapitel 5 bilden: Tobias B u 1 a n g behandelt „Apodiktische Setzung und narrative Perspektivierung. Die Lehre vom Haushaben in Wittenwilers Ring“ und Simon Z e i s b e r g „Bacon in Braubach oder Das Nicht-Wissen des Gelehrten vom Reichwerden. Johann Balthasar Schupps satirische Dissertation De Arte Ditescendi (1648)“. An der Themensetzung ist klar erkennbar, dass Literatur in lateinischer (oder griechischer) Sprache in den wenigsten Fällen im Vordergund steht. Doch in den meisten Beiträgen ist antikes Gedankengut, sind ökonomische Auffassungen, die bis in die Antike zurückreichen - und damit verbunden eine Fülle von facheinschlägigen Texten -, präsent und prägend. Hierarchische Strukturen (wie im Beitrag von Anita T r a n i n g e r) gehen auf dominus und servus zurück, auch wenn nun von „Herr und Knecht“ die Rede ist - und diese (Rück)bezüge und Transformationen werden auch explizit hergestellt. Der Concordia-Diskurs (in Rüdiger S c hn e 11s Eröffnungsbeitrag) enthält eine Vielfalt von Zitaten aus Columella. Simon Z e i s b e r g s Abschlussbeitrag ist zur Gänze einem neulateinischen Text gewidmet, den bisher wahrscheinlich kaum jemand kannte, der aber fest in das reale Leben der Menschen zur Entstehungszeit der Abhandlung eingebettet ist. Einem Trend der Neulateinischen Studien folgt „Antikerezeption und -revision“, wenn Claudia O p i t z - B e l a k h a 1 antike Reflexe in volkssprachlicher Literatur beleuchtet. Sämtliche dramenbasierten Beiträge sind ohne antikes Substrat undenkbar. Das Haus schreiben ist eine Fundgrube für jede/n an antiker Literatur Interessierte/n mit einem ausgeprägt komparatistischem Zugang. Ein spürbarer Mehrwert ergibt sich aus dem oft beschworenen „Sitz im Leben“, der hier in besonderer Intensität, genre- und epochenübergreifend, zum Ausdruck kommt. 
Andrea Beyer, Das Lateinlehrbuch aus fachdidaktischer Perspektive. Theorie, Analyse, Konzeption. Heidelberg: Universitätsverlag Winter 2018. 419 S. ISBN 978-3-8253-6971-1

Die im Rahmen einer Promotion entstandene Arbeit schließt eine Lücke in der fachdidaktischen Forschung: Offensichtlich fehlte es bis dato an einer umfassenden, fächerübergreifenden theoretischen Darstellung zur Konzeption von Lehrbüchern ebenso wie an einheitlichen und überprüfbaren Qualitätsstandards derselben. Aus diesem Desiderat leitet die Verfasserin das Ziel ihrer Arbeit ab: Um sich einer Theorie zur Konzeption von (Latein-)Lehrbüchern anzunähern, wird die theoretische Lehrbuchforschung zunächst mit einer umfangreichen Dokumentenanalyse (acht deutscher Lateinlehrbücher) und einer empirischen Studie verknüpft, in deren Anschluss die Ergebnisse ausgewertet und zusammengefasst werden. Auf dieser Basis wird im letzten Abschnitt der Arbeit eine Theorie zur Lehrbuchkonzeption entworfen, die durch eine Handreichung für Lehrbuchentwickler/innen konkretisiert wird.

Teil I (,Theoretische Grundlagen“) besticht durch eine tief gehende Analyse: Fachbegriffe und Definitionen werden zunächst erläutert und für die eigenen Anliegen konkretisiert. B e y e r geht dabei auch auf verschiedene Spannungsfelder (z.B. das Lehrbuch als Lehrer/innenersatz) ein. Besonders hervorzuheben ist dabei, dass die seit Jahrhunderten festgeschriebene Dichotomie von „Grammatikunterricht“ und anschließender „Lektüre- bzw. Literaturphase“ und ihre Zeitgemäßheit hinterfragt wird (42ff.), wobei die Verfasserin hier selbstverständlich von den Gegebenheiten der deutschen Schullandschaft ausgeht. Dennoch erscheint eine Problematisierung überbordenden Grammatikunterrichts bei verhältnismäßig kurzer Literaturphase in jedem Fall durchaus angebracht.

Sehr umfassend werden im Anschluss nicht nur die lateinischen Übersetzungstexte bestehender Schulbücher, sondern auch die deutschen Sachtexte und die inkludierten (Haus)übungen einer Analyse unterzogen. Die Thematik „Originaltext vs. Kunsttext“ in den Lektionen (60ff.) wird dabei ebenso behandelt wie das gesellschaftspolitische Thema der Bildungssprache in Lehrbüchern (67ff.). Auch die (mittlerweile zum Unwort gewordene) „Kompetenz" wird aus unterschiedlichen Blickwinkeln beleuchtet, problematisiert und eine mögliche Neudefinition ihrer einzelnen Facetten entwickelt. Sehr erfreulich sind dabei die Beobachtungen, dass Lateinkompetenz immer auch mit Deutschkompetenz gleichzusetzen ist (108) und dass die Entwicklung von Literaturkompetenz mittlerweile zum Alleinstellungsmerkmal des altsprachlichen Unterrichts geworden zu sein scheint (127). Ausgesprochen erhellend ist dabei auch die anschließende Besprechung und Analyse des Übersetzungsvorgangs selbst, wobei B e y e $r$ auf Grundlage der Linguistik die Wichtigkeit des Verstehensvorganges unterstreicht.

Im Anschluss daran (163ff.) entwirft sie das „Blumenmodell“ als mögliches neues Kompetenzmodell für den Lateinunterricht: Der sog. „Translationskompetenz“, die die einzelnen sprachlichen Kompetenzen miteinander verknüpft und in Beziehung zueinander setzt, kommt dabei die zentrale Bedeutung zu. Ähnlich kleinschrittig bearbeitet B e ye r danach (kulturhistorische) Sachtexte und Lern- bzw. Leistungsaufgaben in Lateinlehrbüchern.

Teil II-IV präsentiert und interpretiert die Daten der empirischen Studie von acht (deutschen) Lehrbüchern. Diese Daten wurden (1.) anhand linguistischer Messverfahren, (2.) anhand von Taxonomien qualitativer Merkmale und (3.) anhand von Nutzerbefragungen (Lehrende und Lernende aus Berlin) gewonnen, eine spannende, wenn auch für Österreich nur bedingt nutzbare Untersuchung. Insgesamt konstatiert die Verfasserin den Lehrwerken (verursacht durch die immer kürzer werdenden Lebenszyklen der Lateinlehrbücher) viele Schwächen in der Tie- 
fenstruktur. Deren Konzeption scheint nämlich nicht vom Kern her theoriegeleitet und wirklich neu entworfen, sondern auf bestehende Konzepte aufgesetzt und ggf. neu adaptiert zu sein.

Die Schlussfolgerungen in Teil V stellen zunächst jene Voraussetzungen dar, die als äußere Rahmenbedingungen auf die Konzeption von Lateinlehrbüchern einwirken. Im Anschluss erläutert Beyer ihre Theorie zur Konzeption von Lehrbüchern (zentrale Bestandteile, Strukturierung, Qualitätskriterien, notwendige Fähigkeiten für Lehrbuchentwickler/innen). Zuletzt wird die Handreichung zur Konzeption eines Lateinlehrbuches vorgestellt. Nach Stichwörtern der einzelnen Rubriken geordnet, findet man hier kurze Handlungsanweisungen. Analog zum vorangegangenen theoretischen Teil unterscheidet B e y e r hier ebenfalls zwischen ebenenspezifischen und lehrbuchübergreifenden Qualitätsstandards. Hier hätte eine alphabetische Reihung zwar noch etwas mehr Übersichtlichkeit bringen können, insgesamt bietet dieser letzte Teil aber eine griffige und praktikable Zusammenfassung bzw. Nutzbarmachung der im Vorfeld erarbeiteten Ergebnisse.

Nina Aringer 